\title{
Article
}

\section{Sliding Contacts in Planetary Magnetospheres}

\author{
Elena Belenkaya *(D) and Igor Alexeev (D) \\ Skobeltsyn Institute of Nuclear Physics (SINP MSU), Lomonosov Moscow State University, \\ 119991 Moscow, Russia; alexeev@dec1.sinp.msu.ru \\ * Correspondence: elena@dec1.sinp.msu.ru
}

check for updates

Citation: Belenkaya, E.; Alexeev, I. Sliding Contacts in Planetary Magnetospheres. Symmetry 2021, 13, 283. https://doi.org/10.3390/ sym 13020283

Academic Editor: Valery S. Lisitsa

Received: 24 December 2020

Accepted: 2 February 2021

Published: 7 February 2021

Publisher's Note: MDPI stays neutral with regard to jurisdictional claims in published maps and institutional affiliations.

\begin{abstract}
In the planetary magnetospheres there are specific places connected with velocity breakdown, reconnection, and dynamo processes. Here we pay attention to sliding layers. Sliding layers are formed in the ionosphere, on separatrix surfaces, at the magnetopauses and boundaries of stellar astrospheres, and at the Alfvén radius in the equatorial magnetosphere of rapidly rotating strongly magnetized giant planets. Although sliding contacts usually occur in thin local layers, their influence on the global structure of the surrounding space is very great. Therefore, they are associated with non-local processes that play a key role on a large scale. There can be an exchange between different forms of energy, a generation of strong field-aligned currents and emissions, and an amplification of magnetic fields. Depending on the conditions in the magnetosphere of the planet/exoplanet and in the flow of magnetized plasma passing it, different numbers of sliding layers with different configurations appear. Some are associated with regions of auroras and possible radio emissions. The search for planetary radio emissions is a current task in the detection of exoplanets.
\end{abstract}

Keywords: sliding contacts; magnetosphere; planets; exoplanets; reconnection; dynamo

\section{Introduction}

At present, the problem of searching for exoplanets is of relevance in connection with the development of new-generation radio telescopes [1]. The search for coherent cyclotron auroral radio emissions from exoplanets is promising. The most powerful radiation is expected in the presence of a strong magnetic field. Consequently, the study of magnetic fields in the magnetospheres of exoplanets is of increasing importance. The cavity in which the behavior of the plasma is determined by the magnetic field of the planet/exoplanet is called the magnetosphere. The magnetopause is the magnetospheric boundary.

In a star-exoplanet system, the mutual influence of these objects on each other is significant. For exoplanets located close to central stars, their influence on the star is especially important $[2,3]$. There are no such analogues in the solar system. In the interaction of a star-exoplanet, the role of magnetic fields is crucial. In this study we will focus on special locations that are significant in the creation of strong field-aligned currents participating in the generation of auroras [4-6] and in reconnection and dynamo processes [7-10].

Locations where the magnetic field is zero can be neutral points, neutral lines, or neutral surfaces. In the context of the reconnection paradigm in magnetospheric physics, the focus is on the neutral (or quasi-neutral) lines and on neutral points. In the first case there is two-dimensional merging, and in the second case there is three-dimensional reconnection. In the perpendicular cross-section in each point of quasineutral line there is an X-type neutral point like for the neutral line, but the non-zero magnetic field component can exist along the quasi-neutral line. Modeling calculations performed by Belenkaya [11] confirmed the well-known fact that the main factor determining reconnection in the magnetosphere is the direction of interplanetary magnetic field (IMF). Moreover, Belenkaya [11] showed that the presence of the magnetopause current sheet separating solar wind and magnetospheric fields and plasma flows leads to appearance of different types of reconnection in the magnetosphere depending on the direction of the IMF. The paraboloid model of the terrestrial 
magnetospheric magnetic field [12,13] was used by Belenkaya [11]. It was shown that all directions of the IMF can be divided into four cones. For one of them, a two-dimensional (2D) reconnection on the quasi-neutral line occurs; for the second, three-dimensional (3D) merging in two neutral points takes place; and for the third and fourth cones there is one neutral point in one cusp and a neutral (quasi-neutral) line at the magnetopause of the other cusp. For the latter two cases the vertical component of IMF is small, and in which cusp there is neutral point or line depends on the sign of the radial component of IMF.

In the immediate vicinity of the magnetic null near the magnetopause even a very weak IMF becomes comparable with the magnetospheric field. Therefore, in these regions a small IMF can change the global magnetospheric structure of a strongly magnetized planet. Such places associated with plasma sliding and violation of the frozen-in conditions can affect plasma and fields over great distances. Similar non-local phenomena are also typical for planets in the astrospheres of other stars-regions of space around stars in which their stellar winds play a dominant role.

Magnetospheric magnetic field paraboloid models were constructed in our laboratory for the magnetic planets of the solar system: Mercury [14], Earth [15], Jupiter [16], and Saturn [17]. These are currently the most complete analytical models, with a small number of parameters that have the clear physical sense describing the main magnetospheric current systems: the planetary magnetic field, the tail current system, shielding currents at the magnetopause, magnetodisk or ring currents, and partially penetrating IMF. Numerical calculations using these models for different conditions in the solar wind allowed us to interpret observational data for the studied planetary magnetospheres and even construct the transient magnetospheric current systems arising in response to the sharp changes in the solar wind and IMF parameters [18,19]. As a result, these models can be considered as variants of a generalized magnetospheric magnetic field model, which may also be appropriate for exoplanet research [20]. Different elements of the paraboloid magnetospheric magnetic field model can be considered separately under appropriate conditions and refined using new experimental data (e.g., [21]).

Taking into account the peculiarities of each magnetosphere and using observational data from the spacecraft missions we can adequately describe the main processes in planetary environments. The magnetic field structure determines plasma flows for the magnetized plasma. On the sliding layers the frozen-in conditions are violated, and plasma behavior changes in the global scale. Modeling calculations show that IMF plays a crucial role in the large-scale magnetospheric magnetic field, and in this regard the locations and even the number of sliding contacts can change.

Depending on the conditions in the magnetosphere of the exoplanet and in the flow of magnetized plasma passing by it, a different number of slippage layers with different configurations appear. In consideration of this issue, we will start with examples of the planets of the solar system and the heliosphere.

\section{The Earth}

The first sliding contact in the Earth's magnetosphere is located in the atmosphere/ionosphere, where partial slowdown of rotation occurs due to collisions of ions with neutrals participating in rotation with the planet ("friction"). If the angular speed of the Earth's rigid rotation is $\Omega_{\mathrm{pl}}$, then the angular plasma velocity in the upper atmosphere/ionosphere and beyond is $\Omega_{\mathrm{i}} \leq \Omega_{\mathrm{pl}}$. However, the magnetosphere-ionosphere linking influences this effect. For example, on the dawn side of the terrestrial magnetosphere the return convection flow from the night to the dayside adds to the corotation, resulting in supercorotation. Similarly, supercorotating flows on the dawn side of Saturn's magnetosphere has at times been observed by Cassini, and also leads to supercorotation in the corresponding zones of the ionosphere [22]. It was mentioned by the authors that this effect is random and atypical for Saturn. On the duskside of the Earth's magnetosphere the return convection and corotation are antiparallel. Thus, subcorotation is observed in the corresponding ionospheric zones. For southward IMF on the flanks of the Jupiter's magnetosphere there are 
tailward flows, which are parallel to corotation on the dusk and antiparallel on the dawn side (Figures 3 and 7 in [23]). The corresponding zones in the Jovian ionosphere match super- and subcorotation. The calculations in [23] were fulfilled in the Jovian paraboloid magnetospheric model [16]. In any case, velocity changes on the ionospheric level, and we consider this as a sliding contact.

During reconnection of the magnetospheric magnetic field with the interplanetary magnetic field, magnetic field lines of various topological types (closed, open, and interplanetary), separated by separatrix surfaces, appear [24]. Strong field-aligned currents flow along these separatrix surfaces. Each type of field lines corresponds to its own electric field; accordingly, both jumps of electric fields and jumps of plasma velocity can appear on their boundary surfaces, which can be considered as sliding contacts. Since the field lines of the separatrix surfaces intersect at neutral points of the magnetic field, projecting into polar ovals, the field-aligned currents along the separatrix surfaces are sources of discrete auroral emissions, including radio emissions. It is supposed that the auroral emissions are caused by the accelerated magnetospheric electrons in the field-aligned currents flowing from the ionosphere.

A simple scheme of reconnection of a dipole field, simulating the planet's own magnetic field, with a uniform magnetic field of the solar wind is shown in Figure 1. Open field lines form northern and southern tubes bounded by the separatrix surfaces $A_{1}$. Closed dipole-like field lines are placed near the planet and are bounded by the separatrix surfaces $A_{2}$. The surfaces of magnetic field lines $A_{1}$ and $A_{2}$ are connected in the neutral points of the magnetic field $O_{\mathrm{N}}$ and $O_{\mathrm{S}}$. Singular magnetic field lines $B_{1}$ and $B_{2}$ are perpendicular to the separatrix surfaces in magnetic nulls. $B_{2}$ lines intersect the ionosphere in the cusp projections [25].

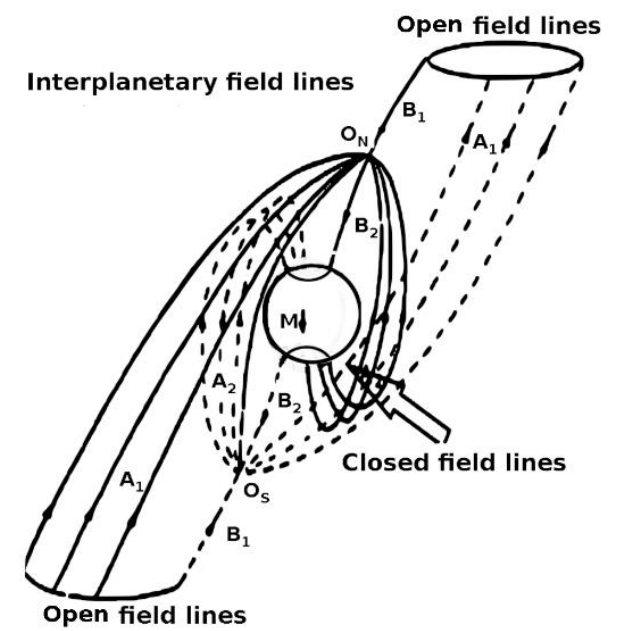

Figure 1. Reconnection of the terrestrial dipole field with interplanetary magnetic field having a northward component (antiparallel to the planetary magnetic dipole axis $M$ directed southward). The scheme is based on Figure 1 from [19] and Figure 6 from [26].

From Figure 1 it follows that for northward IMF with arbitrary radial and azimuthal components all magnetospheric field lines intersect the equatorial magnetosphere. In contrast, for southward IMF (with arbitrary radial and azimuthal components), open field lines do not intersect the equatorial magnetosphere and go to the magnetopause of the corresponding hemispace [27].

In the symmetric structures of a strictly southward or strictly northward IMF, we have two degenerate cases. In the first case a neutral line arises in the equatorial plane of the magnetosphere. It is projected onto two polar ovals in the northern and southern hemispheres. The size of a polar cap (the region inside the oval) is at a maximum in this case. In the second case a closed magnetosphere with a single open field line in each hemisphere coinciding with the dipole axis is formed. The polar cap size is equal to zero. 
Auroral radio emissions (AREs) of the Earth are recorded in the $\mathrm{km}$ range from 100 to $1000 \mathrm{kHz}$ with a maximum at a frequency of $\sim 250 \mathrm{kHz}$ [28]. Accelerated electrons, moving from the plasma sheet of the tail, arising during reconnection, are the source of radio emission. AREs on Earth correlate with ultraviolet (UV) discrete auroral arcs, including transpolar arcs (see [28] and references therein). Zarka [28] noted that a sufficiently large ARE power requires a highly efficient generation mechanism. Up to $\geq 1 \%$ of the power of precipitating electrons goes towards dissipation in the form of radio waves. Hence, it was concluded that it makes sense to consider only coherent processes. Among them, the most promising is the cyclotron-maser instability. It makes it possible to obtain radio emissions at a frequency close to the electron gyrofrequency, which should be higher than the electron plasma frequency of the medium.

\section{Jupiter}

The first sliding contact in the magnetosphere of Jupiter, like that of the Earth, is the ionosphere, where partial deceleration of plasma rotation occurs due to collisions of ions with neutrals. If $\Omega_{\mathrm{pl}}=1.76 \times 10^{-4} \mathrm{rad} \mathrm{s}^{-1}$ is the angular velocity of the planet's rotation, the angular velocity in the upper atmosphere/ionosphere $\Omega_{\mathrm{i}} \leq \Omega_{\mathrm{pl}}$. As mentioned above, the magnetosphere-ionosphere interaction can lead to super-and subcorotation in the different zones of the Jovian ionosphere. Moreover, due to the action of neutral winds moving the plasma by collisions, the generation of electric fields and transfer of momentum the plasma in the ionosphere can be in super- and subcorotation regimes. In any case, as in the case of the Earth, the velocity changes in Jupiter's ionosphere.

The second partial deceleration of plasma occurs in the equatorial magnetosphere at the Alfvén radius. The Alfvén radius is the distance from the center of the planet at which the magnetic energy density is equal to the kinetic energy density of the plasma. At this distance, the speed of rotation of Jupiter's magnetospheric plasma is equal to the Alfvén speed, i.e., the Alfvén Mach number is 1 . The Alfvén radius is located in the equatorial magnetosphere at a distance from the center of the planet from $\sim 18$ to $30 R_{\mathrm{J}}$, where $R_{\mathrm{J}}$ is the radius of Jupiter $\left(R_{\mathrm{J}}=7.1 \times 10^{7} \mathrm{~m}\right)$. Beyond the Alfvén radius, under the action of centrifugal force, magnetic field lines loaded with Io's additional plasma in the lowlatitude magnetosphere are extended into a disk-like structure. Io is located at a distance of $\sim 5.9 R_{\mathrm{J}}$ from the center of Jupiter and due to volcanic activity triggered by tidal forces emits neutrals with a rate $\sim 1 \mathrm{~T} \mathrm{~s}^{-1}$, from which sulfur and oxygen ions arise after ionization. Due to the fast rotation of Jupiter and the presence of a source of inter-magnetospheric plasma (Io), Jupiter's magnetodisk is formed [29-31]. Beyond the Alfvén radius, the plasma dictates the behavior of the magnetic field, while up to the Alfvén radius, the magnetic field controls the plasma motion.

Behind the Alfvén radius, under the action of centrifugal force (which exceeds the force of gravity at a distance of $\sim 2 R_{\mathrm{J}}$ ), a radial expansion of the plasma occurs. As it follows the conservation of momentum, the azimuthal velocity decreases in this case, i.e., the rigid corotation of the magnetospheric plasma partially breaks down; its angular velocity is $\omega<\Omega_{\mathrm{i}}$. This occurs on the field lines crossing the magnetodisk on the distance $\geq$ Alfvén radius, while on the field lines, which do not cross the magnetodisk, there is no such kind of braking.

A partial deceleration of plasma in the equatorial magnetosphere at the Alfvén radius (where unipolar inductor acts), generates strong field-aligned currents, which flow out of the northern and southern ionosphere. These field-aligned currents are associated with auroras in Jupiter's main polar ovals $[29,30]$. These are the brightest, almost constantly present elements of Jupiter's auroras. The brightness in the visible and UV ranges is of the order of $100 \mathrm{kR}$ at a width of $\sim 1000 \mathrm{~km}$ (in latitude about $1^{\circ}$ ) [30] with a peak in the thousand $\mathrm{kR}$ at a width of several hundred $\mathrm{km}[32,33]$. Thus, the source of energy of the auroras in the main ovals of Jupiter is the rotation of the planet. A radial current in the magnetodisk spreads from the field-aligned currents flowing from the main ovals. It is 
closed, presumably, in the distant magnetodisk by the field-aligned currents flowing into the high-latitude ionosphere.

The Alfvén radius in the equatorial magnetosphere is located between Galilean moons Ganymede and Callisto. Depending on the state of the magnetospheric plasma, in particular, on the volcanic activity of Io, it can move within these limits. Up to the Alfvén radius, the magnetospheric plasma rotates at a sub-Alfvénic speed, so the Galilean satellites located there (Io, Europa, Ganymede) excite Alfvén wings, at the roots of which auroral spots appear in Jupiter's ionosphere. While the auroras from these satellites exist practically all the time, the auroras from Callisto rarely occur when this satellite is located in the sub-Alfvenic zone. In addition to the fact that the auroras from Callisto are rare, they are very weak, and special methods are required to distinguish them against the background of the auroras of the main ovals [34,35]. Bhattacharyya et al. [34] used the coaddition technique and on 24 May 2007 separated a candidate for the subcorotating spot in a footprint of Callisto in Hubble Space Telescope (HST) UV images. The special methods used included dark image subtraction, flat-field correction, and instrumental geometric distortion correction. The authors emphasized that in that time Callisto was placed on the current sheet.

The final breakdown of rotation occurs in the equatorial magnetosphere at the plasmapause (which in the case of Jupiter almost reaches the magnetopause), at the magnetopause, and in the tail. The location of sliding contacts in the Jovian magnetosphere depends on the IMF direction. For the closed model of the magnetosphere (in the absence of IMF) in the paraboloid magnetospheric model [16], the projections of lines of constant ionospheric latitudes and longitudes were calculated. The lines of constant ionospheric latitudes are equipotentials of the corotation electric field [23]:

$$
\boldsymbol{E}_{\text {rig }}=-r \Omega_{\mathrm{i}} \sin \theta \boldsymbol{e}_{\phi} \times \boldsymbol{B},
$$

where $\theta$ is the polar angle from the axis of rotation (colatitude), $\boldsymbol{e}_{\phi}$ is the unit vector in the azimuthal direction, and $\boldsymbol{B}$ is magnetic field. Their projections along the equipotential magnetic field lines onto the equatorial plane of the magnetosphere and into the perpendicular cross-section of the distant tail characterize the rotation of the plasma in the Jupiter's magnetosphere.

A paraboloid model of the Jupiter's magnetosphere $[16,23]$ included the planetary magnetic field, magnetodisk's field, field of the tail current system, screening magnetopause currents field, and partially penetrated IMF. Selected parameters of the paraboloid model of the Jupiter's magnetosphere included the distance to the subsolar magnetopause $R_{\mathrm{SS}}=100 R_{\mathrm{J}}$, the distance to the inner edge of the tail current sheet $R_{2}=65 R_{\mathrm{J}}$, the distances to the outer and inner edges of the magnetodisk $R_{\mathrm{D} 1}=92.07 R_{\mathrm{J}}$ and $R_{\mathrm{D} 2}=18.4 R_{\mathrm{J}}$, respectively, and also the magnetic fields $B_{\mathrm{t}}=-2.5 \mathrm{nT}$, which characterizes the magnetic field of the tail current system at the inner edge of the tail current sheet, and $B_{\mathrm{DC}}=2.5 \mathrm{nT}$, which is equal to the disk's magnetic field at the outer edge of the magnetodisk. The angle of inclination of the magnetic dipole to the $\mathrm{Z}$ axis of the solar-magnetospheric coordinate system $\Psi$ was assumed to be zero [16].

The field lines going into the distant tail, which do not cross the equatorial plane, can be considered open in a closed magnetospheric model [36]. Projections of constant latitudes from $82^{\circ}$ to $90^{\circ}$ go to the distant tail. Plasma on these lines is involved in rotation with the planet. Rotation stops at the distant tail cross-section. This is the third sliding contact.

Jupiter's magnetic moment is northward. For southward IMF, the results of calculations in the paraboloid model of the Jupiter's magnetosphere [23] show that projections of constant latitudes that were in the distant tail for IMF $=0$ now go to the equatorial plane. The Alfvén radius along the magnetic field lines is projected from the equatorial magnetosphere to latitudes $\pm 74^{\circ}$, where the main ovals are located [23].

The potential difference generated by a unipolar inductor is $354 \mathrm{MV}$, while the potential difference created by the solar wind through the polar cap is $660 \mathrm{kV}$, with a penetration coefficient of IMF into the magnetosphere $k \sim 0.1$ and of the order of several MV for $k \sim 1$. Thus, the energy, going to the generation of Jupiter's auroras is drawn mainly from the 
rotation of the planet and, to a lesser extent, from the solar wind. At the magnetopause of the opposite hemispace, the final breakdown of rotation on the open field lines occurs (third sliding contact). From the structure of the field lines in the noon-midnight meridional section it follows that in the case of the southward IMF, all field lines intersect the equatorial magnetosphere [23] (as in the case of Earth for northward IMF). Therefore, a partial deceleration of the plasma should occur on them beyond the Alfvén radius.

A different situation arises in the noon-midnight meridional section of Jupiter's magnetosphere at the northward IMF [23]. In this case, the electric field created by the rotation of Jupiter is transmitted along the open field lines into the outer magnetosphere, causing the plasma to rotate with an angular velocity $\Omega_{\mathrm{i}}$. The Alfvén velocity outside the equatorial magnetosphere is very high; in the tail lobes it reaches $\sim 31 \times 10^{3} \mathrm{~km} \mathrm{~s}^{-1}$, so the Alfvén radius may be behind the magnetopause, and rotation can be transmitted to the magnetopause, where it finally stops.

Regardless of IMF, Jupiter's main polar ovals lie in the roots of closed field lines. If for the southward IMF all magnetic field lines intersect the equatorial magnetosphere, where partial deceleration of rotation occurs beyond the Alfvén radius, for the northward IMF there is an open field line tubes going to the magnetopause, without intersection of the equatorial plane, and carrying out rotation there (similar to rotation in the distant tail in a closed magnetospheric model). Rotation finally stops at the magnetopause of the corresponding hemispace; this is the third sliding contact. Thus, the magnetic field of the solar wind determines the number and location of sliding contacts in Jupiter's magnetosphere.

In addition to the auroras in the main ovals and auroral spots in the roots of the Alfvén wings from the Galilean satellites, which draw energy from the corotation of the magnetospheric plasma, there are also auroras in the polar caps caused by the magnetohydrodynamic (MHD) solar wind generator. They are more time-variable. As shown above, all these auroras are associated with sliding contacts.

\section{Dynamo on the Magnetopause of a Rapidly Rotating Magnetic Planet with a Stellar} Wind Magnetic Field Oriented Close to the Magnetic Moment of the Planetary Dipole

For a rapidly rotating planet with a magnetic dipole oriented close to the interplanetary magnetic field, the rotation could be transferred from the planet to the magnetopause, as in the case of Jupiter [7,8]. Under such conditions, the plasma on open field lines, after partial deceleration in the ionosphere, is finally decelerated at the magnetopause. It was shown $[7,8]$ that in this case, behind the magnetopause, the magnetic field can be enhanced by a factor of 10 by the dynamo mechanism. This scenario can also be realized for rapidly rotating magnetic exoplanets. In the dynamo process, the rotational energy is converted behind the magnetopause into the energy of the magnetic field.

The hydromagnetic dynamo is one of the main elements of dynamo theory [37-39]. It implies that under certain conditions, weak magnetic fields are amplified and maintained by the energy of the plasma motion. The main dynamo prerequisites are: three-dimensionality; the interaction of poloidal and toroidal magnetic fields; the presence of dissipation; and the predominance of kinetic energy over magnetic energy in the dynamo region. We suggest that the dynamo acts in a thin layer outside the magnetopause of a rapidly rotating magnetic planet, where the plasma rotation speed gradually breaks down. The non-ideal interaction of the passing plasma flow with the corotating plasma can lead to an increase in the magnetic field of the solar/stellar wind behind the magnetopause.

Thus, if a magnetic exoplanet in the stellar wind with a magnetic field close to its dipole moment rotates fast enough and its ionospheric conductivity is high enough, the planet's rotation is transmitted to the magnetosphere. Along highly conductive open field lines, rotation can be transferred to the magnetopause, outside of which it decays (the Alfvén radius on open field lines can be located behind the magnetopause). The effective conductivity of the magnetopause should be high, but less than the field-aligned conductivity.

For the dynamo to work, a deviation from axial symmetry is required. The damped rotation of the plasma outside the magnetopause pulls the toroidal field $B_{\mathrm{T}}$ out of the weak 
background poloidal field of the stellar wind $B_{\mathrm{P}}$ (this is the so-called $\Omega$-dynamo). The plasma flows of the magnetosheath interacting with the rotating plasma create a nonzero helicity density:

$$
h=V \cdot[\nabla \times V] \neq 0,
$$

proportional to the coefficient $\alpha$ connecting the average emf with $B_{\mathrm{T}}$ and providing the $\alpha$-effect ( $V$ is velocity). The $\alpha$-effect regenerates the poloidal field from the toroidal one and closes the $\alpha \Omega$ dynamo cycle: $\boldsymbol{B}_{\mathrm{P}} \rightarrow \boldsymbol{B}_{\mathrm{T}} \rightarrow \boldsymbol{B}_{\mathrm{P}}$.

Factors lowering the mirror symmetry of the velocity field with respect to the planes perpendicular to the vector $\Omega_{\mathrm{i}}$ include Coriolis forces. Factors breaking the axial symmetry include the presence of meridional velocity components in the flowing stream, and the difference of orientations of the planet's axis of rotation with its magnetic dipole and with the direction of the stellar wind magnetic field. Thus, for magnetic, rapidly rotating exoplanets with a magnetic dipole close to the magnetic field of the stellar wind, sliding contacts at the magnetopause can lead to an increase in the magnetic field of the passing magnetized plasma flow.

\section{Dynamo Behind the Astrosphere of a Rapidly Rotating Magnetic Star}

The dynamo mechanism proposed for the magnetopause of a rapidly rotating magnetic planet was applied to the heliopause $[9,10]$. The Voyager 1 spacecraft crossed the heliopause on 25August 2012 at a distance of 121.7 AU. It was found that the magnetic field doubled (from 0.2 to $0.4 \mathrm{nT}$ ) without changing its direction [40]. Evidence that Voyager 1 entered the interstellar medium was provided by a simultaneous decrease in the number of heliospheric particles and an increase in the intensity of galactic cosmic rays. In addition, the plasma density increased by an order of magnitude, while the temperature decreased. In the works of Belenkaya $[9,10]$, it was shown that all the necessary conditions for the operation of the dynamo were met behind the heliopause. Therefore, this mechanism can explain the observed two fold enhancement of the magnetic field without changing its direction when Voyager 1 entered the interstellar medium.

A spiral magnetic field was observed both inside the heliopause and behind it [41]. The magnetic field of the solar wind is associated with the heliospheric plasma, and the field of the interstellar medium is associated with its plasma. The performed estimates showed that the enhancement of the magnetic field behind the heliopause by the dynamo mechanism is possible in the region containing the point of intersection of the Voyager 1 spacecraft with the heliosphere boundary $[9,10]$.

Similarly, outside the astrosphere of a rapidly rotating magnetic star, an increase in the magnetic field of the magnetized plasma flow bypassing it is possible. This phenomenon may be essential for the amplification of radio emissions.

\section{Conclusions}

The present paper summarizes our ideas based on much modeling and theoretical work undertaken by us earlier, and introduces a new concept of sliding contacts, which are places where plasma velocity changes rapidly. These specific places are associated with the generation of field-aligned currents, energy exchanges, reconnection, and dynamo actions. This concept makes it possible to consider the corresponding non-local processes in relation to different cosmic objects: magnetospheres of planets and exoplanets, heliosphere and astrospheres, etc. Strong field-aligned currents are associated with precipitation of accelerated electrons to the auroral regions with the generation of radio emissions, which is significant for future exoplanetary investigations.

Our analysis of Galilean satellites shows that the Alfvén radius in the Jovian equatorial magnetosphere is located between Ganymede and Callisto depending on the temporal conditions, and beyond it no obstacle in the magnetospheric plasma can form Alfvenic wings with corresponding bright spots in the Jupiter's ionosphere. A similar situation arises for close-in exoplanets. Depending on the location of exoplanet inside the Alfvén 
radius or beyond it, Alfvenic wings or the bullet-form magnetosphere arise. If the stellar wind from the host star is very dense, the Alfvén radius can be very short.

For rapidly rotating magnetic exoplanets with a magnetic dipole oriented close to the magnetic field of the stellar wind, sliding contact at the magnetopause can enhance the magnetic field of the passing magnetized plasma flow.

It was shown by Belenkaya $[9,10]$ that dynamo can work at the heliopause, amplifying magnetic field value just out of it. This suggestion was supported by observations of heliopause intersection by the Voyager 1 spacecraft and later by Voyager 2. Similarly, beyond the boundary of the astrosphere of a rapidly rotating magnetic star, an increase in the magnetic field of the magnetized plasma of the interstellar medium flowing around it is possible, if the interstellar magnetic field orientation is close to the stellar dipole moment direction. Both these phenomena may be essential for the amplification of radio emissions.

Author Contributions: Conceptualization, E.B., I.A.; methodology, E.B.; validation, E.B., I.A.; analysis, E.B.; investigation, E.B., I.A.; writing-original draft preparation, E.B.; writing-review and editing, E.B.; I.A.; visualization, E.B.; supervision, E.B.; project administration, E.B. All authors have read and agreed to the published version of the manuscript.

Funding: This research received no external funding.

Institutional Review Board Statement: Not applicable.

Informed Consent Statement: Not applicable.

Data Availability Statement: Not applicable.

Conflicts of Interest: The authors declare no conflict of interest.

\section{References}

1. Zarka, P.; Le, D.; Grießmeier, J.-M.; Lamy, L.; Girard, J.N.; Hess, S.L.G.; Lazio, T.J.W.; Hallinan, G. Detecting exoplanets with FAST? Res. Astron. Astrophys. 2019, 19, 23. [CrossRef]

2. Ip, W.-H.; Kopp, A.; Hu, J.-H. On the star-magnetosphere interaction of close-in exoplanets. Astrophys. J. Lett. 2004, 602, L53-L56. [CrossRef]

3. Gurumath, S.R.; Hiremath, K.M.; Ramasurbramanian, V. Influence of Planets on the Magnetic Activity of Sun-Like Stars, Long-Term Datasets for the Understanding of Solar and Stellar Magnetic Cycles Proceedings IAU Symposium No. 340; International Astronomical Union, Cambridge University Press: Cambridge, UK, 2018. [CrossRef]

4. Cowley, S.W.H.; Morelli, J.P.; Lockwood, M. Dependence of convective flows and particle precipitation in the high-latitude dayside ionosphere on the $X$ and $Y$ components of the interplanetary magnetic field. J. Geophys. Res. 1991, 96, 5557-5564. [CrossRef]

5. Tanaka, T.; Watanabe, M.; Den, M.; Fujita, S.; Ebihara, Y.; Kikuchi, T.; Hashimoto, K.K.; Kataoka, R. Generation of field-aligned current (FAC) and convection through the formation of pressure regimes: Correction for the concept of Dungey's convection. J. Geophys. Res. 2016, 121, 8695-8711. [CrossRef]

6. Borovsky, J.E.; Birn, J.; Echim, M.M.; Fujita, S.; Lysak, R.L.; Knudsen, D.J.; Marghitu, O.; Otto, A.; Watanabe, T.; Tanaka, T. Quiescent Discrete Auroral Arcs: A Review of Magnetospheric Generator Mechanisms. Space Sci. Rev. 2020, 216, 1-39. [CrossRef]

7. Belenkaya, E.S. The role of planetary rotation in the generation of a magnetic field in a beam of open field lines in the vicinity of the magnetopause. Geomag. Aeron. 1993, 33, 11-16.

8. Belenkaya, E.S. Generation of the magnetic field at the magnetopause of the rapidly rotating planet. J. Geophys. Res. 1996, 101, 26487-26494. [CrossRef]

9. Belenkaya, E.S. Dynamo in the outer heliosheath: Necessary conditions. Sol. Phys. 2015, 290, 2077-2092. [CrossRef]

10. Belenkaya, E.S. Planetary magnetopause and heliopause current sheets. In Electric Currents in Geospace and Beyond, Geophysical Monograph, 1st ed.; Keiling, A., Marghitu, O., Wheatland, M., Eds.; John Wiley \& Sons, Inc.: Hoboken, NJ, USA, 2018; p. 10. ISBN 978-1-119-32449-2.

11. Belenkaya, E.S. Reconnection modes for near-radial interplanetary magnetic field. J. Geophys. Res. 1998, 103, 26487-26494. [CrossRef]

12. Alexeev, I.I.; Shabansky, V.P. A model of a magnetic field in the geomagnetopshere. Planet. Space Sci. 1972, 20, 117-133.

13. Alexeev, I.I. The penetration of interplanetary magnetic and electric fields into the magnetosphere. Journ. Geomagn. Geoelectr. 1986, 38, 1199-1221. [CrossRef]

14. Alexeev, I.I.; Belenkaya, E.S.; Bobrovnikov, S.Y.; Slavin, J.A.; Sarantos, M. Paraboloid model of Mercury's magnetosphere. J. Geophys. Res. 2008, 113, A12210. [CrossRef] 
15. Alexeev, I.I.; Belenkaya, E.S.; Bobrovnikov, S.Y.; Kalegaev, V.V. Modelling of the electromagnetic field in the interplanetary space and in the Earth's magnetosphere. Space Sci. Rev. 2003, 107, 7-26. [CrossRef]

16. Alexeev, I.I.; Belenkaya, E.S. Modeling of the Jovian Magnetosphere. Annal. Geophys. 2005, 23, 809-826. [CrossRef]

17. Belenkaya, E.S.; Cowley, S.W.H.; Meredith, C.J.; Nichols, J.D.; Kalegaev, V.V.; Alexeev, I.I.; Barinov, O.G.; Barinova, W.O.; Blokhina, M.S. Magnetospheric magnetic field modelling for the 2011 and 2012 HST Saturn aurora campaigns-Implications for auroral source regions. Ann. Geophys. 2014, 32, 689-704. [CrossRef]

18. Belenkaya, E.S.; Alexeev, I.I.; Clauer, C.R., Jr. Field-aligned current distribution in the transition current system. J. Geophys. Res. 2004, 109, A11207. [CrossRef]

19. Belenkaya, E.S.; Alexeev, I.I.; Clauer, C.R. Magnetic Field of the Transition Current System: Dawn-Dusk Asymmetry. Ann. Geophys. 2007, 25, 1899-1911. Available online: http://www.ann-geophys.net/25/1899/2007/ (accessed on 5 February 2021). [CrossRef]

20. Belenkaya, E.; Alexeev, I.; Khodachenko, M.; Panchenko, M.; Blokhina, M. Stellar Wind Magnetic Field Influence on the Exoplanet's Magnetosphere; EPSC Abstracts; EPSC2010-72; European Planetary Science Congress: Rome, Italy, 2010 ; Volume 5.

21. Pensionerov, I.A.; Alexeev, I.I.; Belenkaya, E.S.; Connerney, J.E.P.; Cowley, S.W.H. Model of Jupiter's Current Sheet With a Piecewise Current Density. J. Geophys. Res. 2019, 124, 1843-1854. [CrossRef]

22. Masters, A.; Thomsen, M.; Badman, S.V.; Arridge, C.; Young, D.; Coates, A.J.; Dougherty, M.K. Supercorotating return flow from reconnection in Saturn's magnetotail. Geophys. Res. Lett. 2011, 38. [CrossRef]

23. Belenkaya, E.S. The Jovian magnetospheric magnetic and electric fields: Effects of the interplanetary magnetic field. Planet. Space Sci. 2004, 52, 499-511. [CrossRef]

24. Cowley, S.W.H. Earth's Plasma Environment: Magnetic Reconnection and Its Effect on Magnetospheric Fields and Flows. Philos. Trans. Phys. Sci. Eng. 1994, 349, 237-247. Available online: http://www.jstor.org/stable/54256 (accessed on 5 February 2021).

25. Cowley, S.W.H. A qualitative study of the reconnection between the Earth's magnetic field and an interplanetary field of arbitrary orientation. Radio Sci. 1973, 8, 903-913. [CrossRef]

26. Alexeev, I.I.; Belenkaya, E.S.; Bobrovnikov, S.Y.; Kalegaev, V.V.; Cumnock, J.A.; Blomberg, L.G. Magnetopause mapping to the ionosphere for northward IMF. In Annales Geophysicae; European Geosciences Union: Munich, Germany, 2008; Volume 25, pp. 2615-2625.

27. Siscoe, G.L.; Erickson, G.M.; Sonnerup, B.U.O.; Maynard, N.C.; Siebert, K.D.; Weimer, D.R.; White, W.W. Global rope of E Ein magnetopause reconnection: An explicit demonstration. J. Geophys. Res. 2001, 106, 13015-13022. [CrossRef]

28. Zarka, P. Auroral radio emissions at the outer planets: Observations and theories. J. Geophys. Res. 1998, 103, $20159-20194$. [CrossRef]

29. Cowley, S.W.H.; Bunce, E.J. Origin of the main auroral oval in Jupiter's coupled magnetosphere-ionosphere system. Planet. Space Sci. 2001, 49, 1067-1088. [CrossRef]

30. Cowley, S.W.H.; Bunce, E.J.; Nichols, J.D. Origins of Jupiter's main oval auroral emissions. J. Geophys. Res. $2003,108,8002$. [CrossRef]

31. Nichols, J.D.; Cowley, S.W.H. Magnetosphere-ionosphere coupling currents in the Jupiter's middle magnetosphere: Effect of precipitation induced enchancement of the ionospheric Pedersen conductivity. Ann. Geophys. 2004, 22, 1799-1827. [CrossRef]

32. Prange, R.D.; Rego, L.; Pallier, J.E.; Connerney, P.; Zarka, P.; Queinnec, J. Detailed study of FUV jovian auroral features with the post-COSTAR HST faint object camera. J. Geophys. Res. 1998, 103, 20195-20215. [CrossRef]

33. Vasavada, A.R.; Bouchez, A.H.; Ingersoll, A.P.; Little, B.; Anger, C.D. Jupiter's visible aurora and Io footprint. J. Geophys. Res. 1999, 104, 27133-27142. [CrossRef]

34. Bhattacharyya, D.; Clarke, J.T.; Montgomery, J.; Bonfond, B.; Gerard, J.-C.; Grodent, D. Evidence for auroral emissions from Callisto's footprint in HST UV images. J. Geophys. Res. 2018, 123, 364-373. [CrossRef]

35. Belenkaya, E.S. Callisto in the magnetosphere of Jupiter. Sol. Syst. Res. 2020, 54, 85-95. [CrossRef]

36. Alekseev, I.I.; Belenkaya, E.S.; Saybek, D.G. Open field lines in a closed model of the magnetosphere. Geomagn. Aeron. 1998, 38, 9-18.

37. Elsasser, W.M. Background of the geomagnetic dynamo theory. J. Geophys. Res. 1956, 61, 340-347.

38. Moffat, H.K. Magnetic Field Generation in Electrically Conducting Fluids; Cambridge University Press: Cambridge, UK; London, UK; New York, NY, USA; Melbourne, Australia, 1978; p. 343.

39. Yokoi, N. Cross helicity and related dynamo. Geophys. Astrophys. Fluid Dyn. 2013, 107, 114-184. [CrossRef]

40. Burlaga, L.F.; Ness, N.F.; Stone, E.C. Magnetic field observations as Voyager 1 entered the heliosheath depletion region. Science 2013, 341, 147-150. [CrossRef] [PubMed]

41. Burlaga, L. Voyager observations of the magnetic field in the heliosheath and the local interstellar medium. J. Phys. Conf. Ser. 2015, 642, 012003. [CrossRef] 